\title{
FREE TRANSPORT LIMIT FOR $N$-PARTICLES DYNAMICS WITH SINGULAR AND SHORT RANGE POTENTIAL.
}

\author{
J. BARRÉ AND P. E. JABIN
}

\begin{abstract}
We study the limit of systems of interacting particles, when the number of particle becomes very large. The support of the interaction vanishes as the number of particles goes to infinity, so that the natural limit is just free transport, but no limitation is assumed about the strength of the interaction. We obtain explicit estimates for the number of particles effectively interacting and describe the way they do it.
\end{abstract}

\section{INTRODUCTION}

We study the dynamics of many interacting particles in the limit of an infinite number of particles. The force acting on each particle is a sum of pair-wise interaction with the other particles. The kind of dynamics that is expected at the limit depends on the scaling of the force term with respect to the number of particles $N$. Here we consider very short range interaction in the sense that the force between two particles vanishes if their distance is larger than $R$ with, in dimension 3

$$
N R^{2}<<1 \text {. }
$$

In this scaling the formal limit is simply free transport : each particle moves with its initial velocity. Indeed a formal computation easily shows that on average a particle should never undergo a collision : i.e. the number of particles coming at a distance less than $R$ to another is negligible in front of $N$, in a time interval of order 1 .

This is a simplified problem for the more interesting case $N R^{2}=$ const where the obtention of collisional models (of Boltzmann type) is conjectured. The first rigourous step in that direction was obtained by Lanford in a celebrated work [12] (see also [13]). This result nevertheless suffers from two important restrictions : First the particles are hard spheres (they interact with the potential $\Phi(x)=+\infty$ if $|x| \leq R$ and $\Phi=0$ if $|x|>R$ ). And second the limit is only valid for a time

2000 Mathematics Subject Classification. Kinetic theory of gases; Interacting particles systems. 
small with respect to the average time it takes for one particle to have a collision (or the mean free path after rescaling).

This second restriction was improved by Cercignani, Illner and Pulvirenti in [5] (see also [10]); The time interval of validity was still finite and of order the mean free path though. Both results deal with hard spheres although extensions to repulsive potentials are mentioned (see [13]) but unpublished (as far as we know).

Those results are easily extended to our scaling. The limitation on the time interval is no more a real issue as the mean free path tends to infinity. However the proofs would still require hard spheres interaction (or at least a repulsive potential). Note that in the case of an arbitrary potential, the velocity bounds needed to substantiate the intuition behind the formal scaling argument are not easy to prove; in addition, the very concept of collision needs a careful definition.

We start with Prop.2.4 which justifies the free transport limit, still only for repulsive potentials. The interest is mainly in the simplicity of the proof as the result is not really new (although strictly speaking never stated before for a potential with so little regularity).

The main result is Theorem 2.5. It unfortunately deals with an even shorter range $R<<N^{-3 / 5}$ but it assumes virtually nothing on the potential (it does not need to be repulsive and its scale could be as large as one wants) and therefore it is not at all included in previous contributions. Moreover we not only prove the free transport limit but also describe all possible collision sequences between particles and the result is consequently much more precise. The core of the proof is to ensure that the build up of correlations between particles does not destroy the validity of simple scaling arguments. Theorem 2.5 leaves open the question of non trivial limit in the range $N^{-1 / 2}<<R<N^{-3 / 5}$ (for which we have no particular clue to offer). The particles velocities could then become unbounded and the limit would not necessarily be free transport (as the probability of interacting with another particle would increase accordingly).

Finally and for the sake of completeness, let us mention that the limit dynamic is also an important and fertile field of study in the case of long range potentials (and obviously with a weaker scaling). Vlasov-like kinetic equations are then expected. The first rigorous results were obtained by Braun and Hepp [4], Neunzert and Wick [14] and Dobrushin [6], and concern very regular interaction potentials. We also refer to Spohn [17] where the Wasserstein distance is used to obtain the convergence. Two references related to numerical simulations with particles' methods are for instance Victory and Allen [18] and Wollman [19]. All these works require a regular interaction kernel (at 
least continuous and usually even lipschitz). Recently, Hauray and Jabin [9] proved the convergence of a particle approximation to the Vlasov equation for a class of weakly singular potentials. However, the physical potentials of interest are typically the much more singular Coulombian and gravitational interactions, for which no convergence results to the Vlasov equation are known. This difficulty to deal with the short range singularity of potentials in the Vlasov limit was also an incentive for the study of this paper: what happens for a general singular potential in a short range scaling.

\section{Setting And Statement of the Results}

We study the perturbation of the free transport of $N$ point particles by a very short range (but strong) interaction potential. Denote respectively by $X_{i}(t) \in \mathbb{R}^{3}$ and by $V_{i}(t) \in \mathbb{R}^{3}$ the position and velocity at time $t$ of the particle number $i$. The $X^{\prime}$ 's and $V$ 's are governed by the following equations of motion :

$$
\begin{aligned}
& \dot{X}_{i}=V_{i} \\
& \dot{V}_{i}=\frac{1}{S_{N}} \sum_{j \neq i} K_{\varepsilon}\left(X_{i}-X_{j}\right), \quad i=1 \ldots N,
\end{aligned}
$$

where $S_{N}$ is a scaling factor. $S_{N}=N$ is the usual Vlasov scaling; in the following, $S_{N}$ will be $N$ or $1 . K_{\varepsilon}(x)$ is compactly supported in the ball of radius $\varepsilon^{k}$ but singular. It is assumed to be derived from a potential

$$
K_{\varepsilon}=-\nabla_{x} \Phi_{\varepsilon}, \quad K_{\varepsilon}(-x)=-K_{\varepsilon}(x)
$$

This is not strictly necessary and could be replaced by a weaker assumption on the compressibility and energy bounds for the corresponding flow but is generally satisfied by the physical kernels we would wish to use (with some exceptions).

The parameter $\varepsilon$ is directly connected to the number of particles, being the average distance in the phase space between two particles or

$$
\varepsilon=N^{-1 / 6} \text {. }
$$

The initial conditions $X_{i}^{0}$ and $V_{i}^{0}$ are typically random although very large values should be avoided. For simplicity we will consider initial values $I=\left(X_{1}^{0}, V_{1}^{0}, \ldots, X_{N}^{0}, V_{N}^{0}\right)$ in the hypercube $I \in[-1,1]^{6 N}$. In the following, the measure of a set $A$ will always mean the Lebesgue measure, and be denoted by $|A|$. 
Our aim is to compare this dynamics to the unperturbed one, which is simply given by

$$
\frac{d \bar{X}_{i}}{d t}=V_{i} \quad \frac{d \bar{V}_{i}}{d t}=0, \quad i=1 \ldots N
$$

If $S_{N}=N$, this is therefore a kind of mean field limit (but in a weak form) for which much is known provided $K_{\varepsilon}$ is regular enough. Here we only assume the minimal regularity necessary to ensure that the system 2.1 is well defined, that is (see Ambrosio [1] or Hauray for the particular case considered here [8])

$$
K_{\varepsilon} \in B V\left(\mathbb{R}^{3}\right), \operatorname{supp} K_{\varepsilon} \subset B\left(0, \varepsilon^{k}\right)
$$

Note that in addition any other assumption ensuring well posedness of (2.1) for a given $N$ would be enough.

Let us first give some definitions.

Definition 2.1. Two particles are said to collide iff

$$
\exists t \text { s.t. }\left|X_{i}(t)-X_{j}(t)\right| \leq \varepsilon^{k} .
$$

Let us note that we have no information on the duration of a collision; unless otherwise stated, the "time of a collision" is to be understood in the following as the beginning time of the collision, that is the smallest time $t$ such that $\left|X_{i}(t)-X_{j}(t)\right| \leq \varepsilon^{k}$.

Definition 2.2. We denote by $i R_{T} j$ the relation " $i$ and $j$ collide before time $T$ ".

Definition 2.3. A sequence of collisions is an orbit of the relation $R_{T}$.

An easy formal computation tells us that the average number of particles having a collision should be of order $N^{2} \varepsilon^{2 k}$. This number is large (if $k<6$ ) but nevertheless small with respect to $N$ if $k>3$. If this can be rigourously justified, we would therefore obtain, at the limit $N \rightarrow \infty$, the dynamics (2.3) for almost all particles. As all particles are identical, proving this is equivalent to controlling the set of initial conditions for which a chosen particle (the first one for example) has a collision, or

Proposition 2.4. Take $S_{N}=N$. Assume that $\Phi_{\varepsilon} \geq 0$ and $\left|\Phi_{\varepsilon}\right| \leq$ $C /|x|$. For any $6>k>3$ (such that $N \varepsilon^{2 k}<<1$ ), fix a time $T, N$ large enough and consider (2.1) until time $T$. Then the set of initial data I for which the first particle has at least one collision is of measure less than $C N \varepsilon^{2 k}$. 


\section{Remark.}

The two big restrictions are the conditions that the potential be repulsive and that $S_{N}=N$. At first glance and formally, it would seem that they should not be necessary. Contrary to the case of Theorem 2.5 below, here 3-particles collision (or more) can and will typically occur. They are however needed to control the velocities of the particles (in the cases of 3 or more particles collisions). And notice that the formal computation predicting that only $N^{2} \varepsilon^{2 k}$ particles will undergo a collision, only holds if the velocities of the particles remain of order 1 .

This proposition is enough to obtain the limit but it does not describe at all what happens to the particles which have collisions (except that they do not interact with too many other particles) or in the case of attractive (or non repulsive) potentials. If $k$ is large enough, it is possible to be more specific in the sense that two particles may collide only once and a sequence of collisions is exactly that: collisions happening one after the other without overlapping. To be that specific it is unfortunately necessary to exclude the possibility of 3-particles collision. This is automatic for hard spheres, but is requires a more stringent condition if general potentials are included, namely $k>18 / 5$.

More precisely

Theorem 2.5. Take $S_{N}=1$ and any $k>3$ (such that $N \varepsilon^{2 k}<<1$ ). Fix a time $T, N$ large enough and consider (2.1) until time $T$. Then, there exists a large set $O \subset[-1,1]^{6 N}$ with

$$
\left|[-1,1]^{6 N} \backslash O\right| \leq C_{T} N^{3} \varepsilon^{5 k},
$$

such that for all $I \in O$, we have the following

(i) All collisions involve only two particles; that is:

$$
\forall i, j, l, \forall t<T,\left|X_{i}(t)-X_{j}(t)\right|>\varepsilon^{k} \text { or }\left|X_{i}(t)-X_{l}(t)\right|>\varepsilon^{k} .
$$

This allows us to define the number of collisions in a given sequence.

(ii) Two particles belonging to the same sequence at a certain time $t<T$ do not collide again before time $T$.

(iii) Define $O_{n}$ as the subset of $O$ containing the initial data for which there are at least one sequence with exactly $n$ collisions in the dynamics. Then

$$
\left|O_{n}\right| \leq C_{T, n} N^{n+1} \varepsilon^{2 k n}
$$

\section{Remarks.}


(1) Point ( $i$ i ) may be formulated more precisely: consider a collision sequence, and two indices $i$ and $j$ in this sequence undergoing an actual collision, that is $i R_{T} j$. Consider now the relation $R_{T, i j}$, defined as the relation $R_{T}$ excluding $i R_{T} j$, and define $A_{i}$ and $A_{j}$ the orbits of $i$ and $j$ under $R_{T, i j}$. Then $A_{i} \cap A_{j}=\emptyset$. This amounts to say that particles correlated in some way by belonging to the same collision sequence do not collide again.

(2) The theorem is interesting only for $k$ such that $N^{3} \varepsilon^{5 k} \leq 1$ and $n$ large enough such that we have $N^{n+1} \varepsilon^{2 k n}<1$. Note that for $k>3$, there is always a $n$ such that the above quantity vanishes to 0 with $N$ (remember that $N \varepsilon^{6}=1$ ). The first condition however implies that $k \geq 18 / 5$.

Finally let us stress that those two results leave open all the range of cases $3<k<18 / 5$ for general potentials. Of course the assumptions on the potential in Theorem 2.5 are very weak $\left(K_{\varepsilon}\right.$ could even blow up with $N$ ) but we do not know how to proceed even with a precise scaling $\left(S_{N}=N\right.$ for instance).

\section{Proof of Proposition 2.4}

We first need some control on the maximal number of interacting particles.

Denote by $B_{0}$ the set of initial data such that any two particles $i$ and $j$ satisfy initially that

$$
\left|X_{i}(0)-X_{j}(0)\right|>\frac{1}{N}
$$

Clearly, as there is no dynamics yet

$$
\left|[-1,1]^{6 N} \backslash B_{0}\right| \leq \frac{1}{N}
$$

We define a $K$-collision between two particles $i$ and $j$ if there exists $t$ such that $\left|X_{i}(t)-X_{j}(t)\right| \leq K \varepsilon^{k}$.

Denote now by $B_{n}^{K}$ the subset of $B_{0}$ of initial conditions such that there is no sequence of $K$-collisions involving $n+1$ particles or more before time $T$ and by $O_{n}^{K}=B_{0} \backslash B_{n}^{K}$. We will first prove that

$$
\left|O_{n}^{K}\right| \leq C_{n, K} N^{n+1} \varepsilon^{2 k n}
$$

We start with a control on the velocity

Lemma 3.1. For $K \geq 1, I \in B_{n}^{K}$, any $i=1 \ldots N$ and any $t \in[0, T]$, then we have that

$$
\left|V_{i}(t)\right| \leq 1+C n .
$$


Proof: As $I \in B_{n}^{K}$ with $K \geq 1$, the particle $i$ interacts with at most $n$ other particles $j_{1} \ldots j_{n}$. Denote $j_{0}=i$. As $\Phi_{\varepsilon} \leq 0$, we hence obtain by energy conservation that

$$
\sum_{k=0}^{n}\left|V_{j_{k}}(t)\right|^{2} \leq \sum_{k=0}^{n}\left|V_{j_{k}}(0)\right|^{2}+\frac{1}{N} \sum_{0 \leq k \neq m \leq n} \Phi_{\varepsilon}\left(X_{j_{k}}(0)-X_{j_{m}}(0)\right) .
$$

Recalling that $I \in B_{0}$, we know that $\left|X_{j_{k}}(0)-X_{j_{m}}(0)\right|>1 / N$ and as $\left|\Phi_{\varepsilon}\right| \leq C /|x|$, we deduce that

$$
\sum_{k=0}^{n}\left|V_{j_{k}}(t)\right|^{2} \leq 1+n+C n^{2}
$$

hence the result.

Let us now prove by induction the estimate on $\left|O_{n}^{K}\right|$. The initial step and the $n \rightarrow n+1$ step are very similar so we do only the second one. Assume therefore that

$$
\left|O_{n}^{K}\right| \leq C_{n, K} N^{n+1} \varepsilon^{2 k n}
$$

and consider a initial data $I \in O_{n+1}^{K}$. Necessarily there exists $j_{1} \ldots j_{n+1}$ and a time $t_{0}<T$ such that before $t_{0}$ there was no sequence of $K$ collisions involving more than $n+1$ particles and at $t_{0}$ at least another particle, denoted by $i$, starts a $K$-collision with one of the $j_{k}$.

Notice that there could be more than one particle having a collision at $t_{0}$, in which case we just choose anyone of them. Moreover the particle $i$ may have had collisions with another set of particles before $t_{0}$ but with none of the $j_{k}$.

Divide the time interval $[0, T]$ into $M \leq C(1+n) T \varepsilon^{-k}$ small intervals $\left[t_{\alpha}, t_{\alpha+1}\right]$ of size less than $\varepsilon^{k} / 2(1+C n)$.

Through Lemma 3.1, we know that until time $t_{0}$, the velocity of every particle is less than $1+C n$. Therefore choosing $t_{\alpha}=\max \left\{t_{\beta} \mid t_{\beta}<t_{0}\right\}$, we know that at $t_{\alpha}, X_{i}\left(t_{\alpha}\right)$ is at a distance less than $(1+K) \varepsilon^{k}$ from one of the $j_{1} \ldots j_{n+1}$. For the same reason, as $j_{1} \ldots j_{n+1}$ form a sequence of $K$-collisions before $t_{0}$, they also form a sequence of $K+1$-collisions before $t_{\alpha}$.

Consequently denote by $O_{n}^{K}\left(i, j_{1}, \ldots, j_{n+1}, \alpha\right)$ the set of initial data $I \in B_{0}$ such that $j_{1} \ldots j_{n+1}$ form a sequence of $K+1$-collisions before $t_{\alpha}, i$ has no $K$-collision with any of the $j_{k}$ before $t_{\alpha}$ and for some $k=1 \ldots n+1,\left|X_{i}\left(t_{\alpha}\right)-X_{j_{k}}\left(t_{\alpha}\right)\right| \leq(K+1) \varepsilon^{k}$.

Controlling the measure of this set is easy because there is no interaction between particles $j_{1} \ldots j_{n+1}$ and the other particles. Choose first $X_{j_{1}}(0), V_{j_{1}}(0) \ldots X_{j_{n+1}}(0), V_{j_{n+1}}(0)$. The trajectory of the $j_{k}$ particles is then determined. The measure of the set of initial values 
$X_{k}(0), V_{k}(0), k \neq j_{1} \ldots j_{n+1}$ is then less than the measure of the set of initial condition such that at a given time $X_{i}$ is in a given ball of radius $(K+1) \varepsilon^{k}$. By Galilean invariance, this is less than

$$
C(K+1)^{3} \varepsilon^{3 k}
$$

Finally the measure of the set of $X_{j_{1}}(0), V_{j_{1}}(0) \ldots X_{j_{n+1}}(0), V_{j_{n+1}}(0)$ such that they form a sequence of $K+1$-collisions before $t_{\alpha}$ is simply

$$
C_{n, K+1} \varepsilon^{2 n k} \text {. }
$$

Indeed from the induction assumption for the previous step $n$ (but with $K+1$ instead of $K$ ), the measure of the set of initial data such that $n+1$ particles form a sequence of $K+1$-collisions is less than

$$
C_{n, K+1} N^{n+1} \varepsilon^{2 n k} .
$$

As this set is invariant under permutations on the indices of the particles (and as the measure of the set for which two sequences of collisions occur is trivially much lower), we obtain the claimed bound.

Finally

$$
\left|O_{n}^{K}\left(i, j_{1}, \ldots, j_{n+1}, \alpha\right)\right| \leq C C_{n, K+1}(K+1)^{3} \varepsilon^{(2 n+3) k} .
$$

As

$$
O_{n+1}^{K} \subset \bigcup_{i, j_{1}, \ldots, j_{n}=1}^{N} \bigcup_{\alpha=1}^{M} O_{n}^{K}\left(i, j_{1}, \ldots, j_{n+1}, \alpha\right),
$$

we obtain that

$\left|O_{n+1}^{K}\right| \leq N^{n+2} M C C_{n, K+1}(K+1)^{3} \varepsilon^{2(n+1) k+1} \leq C_{n+1, K} N^{n+2} \varepsilon^{2(n+1) k}$,

with

$$
C_{n+1, K}=C C_{n, K+1}(K+1)^{3}(1+n) T .
$$

This proves (3.2).

With (3.2), it is quite straightforward to show Prop. 2.4. Indeed choose $n$ large enough such that

$$
N^{n+1} \varepsilon^{2 k n} \leq N \varepsilon^{2 k} .
$$

It is enough to take $n(k / 3-1) \geq k / 3$ so that $n$ does not depend on $N$. Denote by $D_{1}$ the set of initial data such that the first particle has a collision before time $T$. Denote $\tilde{D}_{1}=D_{1} \cap B_{n}^{1}$. Controlling $\tilde{D}_{1}$ is enough as

$$
\begin{aligned}
\left|[-1,1]^{2 N} \backslash B_{n}^{1}\right| & \leq\left|[-1,1]^{2 N} \backslash B_{0}\right|+\left|B_{0} \cap O_{n}^{1}\right| \leq \frac{1}{N}+C_{n, T} N^{n+1} \varepsilon^{2 k n} \\
& \leq C_{T} N \varepsilon^{2 k}
\end{aligned}
$$

as $k<6$ and $n$ is from now on fixed. 
For any $I \in \tilde{D}_{1}$, there exists $t_{0}$ such that the first particle has no collision until $t_{0}$ and a collision occurs with another particle $i \neq 1$ (possibly more than 1 ) at $t_{0}$. As $I \in B_{n}^{1}$, all velocities are bounded by $1+C n$. Consequently if we again divide $[0, T]$ into $M$ intervals $\left[t_{\alpha}, t_{\alpha+1}\right]$ with $M \leq C(1+n) T \varepsilon^{-k}$, and if we choose $t_{\alpha}=\max \left\{t_{\beta}<t_{0}\right\}$ then $\left|X_{1}\left(t_{\alpha}\right)-X_{i}\left(t_{\alpha}\right)\right| \leq 2 \varepsilon^{k}$.

Denote by $\tilde{D}_{1}(i, \alpha)$ the subset of $B_{n}^{1}$ such that the first particle has no collision before $t_{\alpha}$ and $\left|X_{1}\left(t_{\alpha}\right)-X_{i}\left(t_{\alpha}\right)\right| \leq 2 \varepsilon^{k}$. We have that

$$
\tilde{D}_{1} \subset \bigcup_{i=1}^{N} \bigcup_{\alpha=1}^{M} \tilde{D}_{1}(i, \alpha) \text {. }
$$

As until $t_{\alpha}$ the trajectory of the first particle is a line, it is obvious that

$$
\left|\tilde{D}_{1}(i, \alpha)\right| \leq C \varepsilon^{3 k}
$$

so that

$$
\left|\tilde{D}_{1}\right| \leq C N M \varepsilon^{3 k}=C N \varepsilon^{2 k},
$$

which finishes the proof of Proposition 2.4.

\section{Proof of Theorem 2.5}

The theorem is proved in two steps. First assuming point $(i)$ and (ii) are known, we prove $(i i i)$. We end up with the proof for points $(i)$ and $(i i)$.

4.1. Proof of point (iii) knowing $(i)$ and $(i i)$. In this subsection we assume we have a set $O$ such that for any $I \in O,(i)$ and $(i i)$ of Theorem 2.5 are true.

Proposition 4.1. Let $I \in O$ and $A$ be a sequence with $n$ collisions. Then $\operatorname{card}(A)=n+1$.

Proof : We argue by induction. For $n=1, \operatorname{card}(A)=2$, since one collision involves two, and only two, particles.

We suppose now that the property is true for all $m<n$. Let $i$ and $j$ be the two particles involved in the last collision of the sequence $A$. As above, we define $A_{i}$ and $A_{j}$ the collision sequences of $i$ and $j$ when the collision between $i$ and $j$ is excluded. Then, using Point $(i i)$ of Theorem 2.5, $A_{i} \cap A_{j}=\emptyset$, so that $\operatorname{card}(A)=\operatorname{card}\left(A_{i}\right)+\operatorname{card}\left(A_{j}\right)$. Now, $A_{i}$ and $A_{j}$ are sequences with respectively $m$ and $n-1-m$ collisions. Using the induction hypothesis, we get

$$
\operatorname{card}(A)=m+1+(n-1-m+1)=n+1 .
$$

The proof is completed. 
Let us now study $O_{n}$ the subset of $O$ such that for $I$ in $O_{n}$ the dynamics has at least one sequence with exactly $n$ collisions. Then

Proposition 4.2. Let $n+1$ particles follow the equations of motion:

$$
\left\{\begin{array}{l}
\dot{X}_{i}=V_{i} \\
\dot{V}_{i}=\frac{1}{N} \sum_{j=1, j \neq i}^{n+1} K_{\varepsilon}\left(X_{i}-X_{j}\right)
\end{array}\right\}
$$

We denote by $\omega_{n}$ the measure in $\mathbb{R}^{6(n+1)}$ of the initial conditions such that the $n+1$ particles belong to the same sequence. Then $\left|O_{n}\right| \leq$ $C_{N}^{n+1}\left|\omega_{n}\right|$.

Proof : The argument is very simple. Consider the $n+1$ particles composing the sequence with $n$ collisions in $O_{n}$. There are $C_{N}^{n+1}$ possible choices of indices for those particles but once they are chosen they follow exactly (4.1). Indeed as they belong to a sequence with exactly $n$ collisions, they do not come within $\varepsilon^{k}$ of any other particle.

Their initial positions and velocities therefore belong to $\omega_{n}$ hence the result.

We may now concentrate on the evaluation of $\left|\omega_{n}\right|$. The heuristics behind the next proposition is the following: two particles collide at a given time if they are inside a volume of order $\varepsilon^{3 k}$; otherwise stated, the measure of initial conditions leading to collision at a given time is of order $\varepsilon^{3 k}$, since measure is preserved by the flow. Now, the measure of initial conditions leading to collision at any time is of order $\varepsilon^{2 k}$. The following proposition states that all collisions in a sequence of $n$ brings one such factor $\varepsilon^{2 k}$.

Proposition 4.3. Under the same hypothesis as for Prop. 4.2,

$$
\left|\omega_{n}\right| \leq a_{n, T} \varepsilon^{2 n k}
$$

Point (iii) of Theorem 2.5 immediately follows from this proposition and the previous one.

Three lemmas are necessary for the proof of Prop. 4.3

Lemma 4.4. Take $I \in O$. Let $i$ be a particle belonging to a sequence of $n$ collisions. Then whenever $i$ is not colliding with another particle, $\left|V_{i}\right| \leq \sqrt{n+1}$.

Proof: Energy conservation during a collision between $i$ and $j$ implies that

$$
\left|V_{i}^{\prime}\right|^{2}+\left|V_{j}^{\prime}\right|^{2}=\left|V_{i}\right|^{2}+\left|V_{j}\right|^{2}
$$


where the primes refer to quantities after collision. This equality is valid when the collision is over. Initially, $\left|V_{i}\right|^{2} \leq 1$ for all $i$. Thus after one collision, $\left|V_{i}\right|^{2} \leq 2$ for all $i$, which proves the property for $n=1$.

We assume that the property is true for all $m<n$ and argue by induction.

Consider now any collision in the sequence, involving particles $i$ and $j$; we call $m_{i}$ and $m_{j}$ the number of particles involved in the collision sequences of $i$ and $j$ before the considered collision. Then $m_{i}+m_{j} \leq n-1$. After the collision

$$
\begin{aligned}
\left|V_{i}^{\prime}\right|^{2} & \leq\left|V_{i}\right|^{2}+\left|V_{j}\right|^{2} \\
& \leq m_{i}+1+m_{j}+1 \\
& \leq n+1,
\end{aligned}
$$

using the induction hypothesis.

Lemma 4.5. Take $I \in O$. Let $i$ be a particle belonging to a sequence of $m$ collisions. Then during a time interval $\left[T_{1}, T_{2}\right]$, $i$ travels a distance smaller than $\sqrt{m+1}\left(T_{2}-T_{1}\right)+m \varepsilon^{k}$.

Proof: When $i$ is not colliding, its velocity is bounded by $\sqrt{m+1}$, thanks to Lemma 4.4.

When $i$ is colliding with another particle $j$, it's not possible to bound its velocity, but its position $X_{i}$ can be controlled by tracking $G$, the center of mass of particles $i$ and $j$ : the velocity of the center of mass $V_{G}$ is bounded by $\sqrt{m+1}$, and during the collision, $\left|X_{i}(t)-X_{G}(t)\right| \leq \varepsilon^{k} / 2$. Thus for a collision lasting $\tau$, particle $i$ travels at most a distance $\tau \sqrt{m+1}+\varepsilon^{k}$. Finally, adding the effects of at most $m$ collisions, particle $i$ between $T_{1}$ and $T_{2}$ travels at most $\sqrt{m+1}\left(T_{2}-T_{1}\right)+m \varepsilon^{k}$.

Lemma 4.6. Let $\Omega$ be a bounded region in $\mathbb{R}^{6}$. Consider the dynamics of $n+1$ particles given by (4.1). We denote by $p_{n, T}^{i, \Omega}$ the measure in $\mathbb{R}^{6(n+1)}$ of the initial conditions such that the $n+1$ particles belong to the same sequence, and at time $T,\left(X_{i}(T), V_{i}(T)\right) \in \Omega$. Then $p_{n, T}^{i, \Omega} \leq$ $\left|\omega_{n}\right||\Omega|$.

Proof: This is an easy consequence of the galilean invariance of the dynamics. In other words if $X_{j}, V_{j}, j=1 \ldots n+1$, satisfy (4.1) and belong to the same sequence then for any $x, v \in \mathbb{R}^{3}, X_{j}+x+v t, V_{j}+v$ satisfy again (4.1) and belong to the same sequence.

Proof of Prop. 4.3: We divide the time interval $[0, T]$ in $M$ small intervals of size $\Delta t=\varepsilon^{k} /(2 \sqrt{n+1})$, and consider the times $t_{l}=$ 
$l \varepsilon^{k} /(2 \sqrt{n+1})$, with $l$ an integer. Then

$$
M=\left[2 T \sqrt{n+1} \varepsilon^{-k}\right]+1 \leq 3 T \sqrt{n+1} \varepsilon^{-k},
$$

for $\varepsilon$ small enough. Then if particles $i$ and $j$ collide, there exists $l$ such that

$$
\left|X_{i}\left(t_{l}\right)-X_{j}\left(t_{l}\right)\right| \leq(n+2) \varepsilon^{k}
$$

This is a direct consequence of lemma 4.5.

We call $I_{i j, l}$ the set of initial conditions such that: $n$ collisions happen; $l$ is the smallest integer such that $\left|X_{i}\left(t_{l}\right)-X_{j}\left(t_{l}\right)\right| \leq(n+2) \varepsilon^{k}$; the last collision occurs between particles $i$ and $j$. "Last" means here that at time $t_{l}$, all collisions of the sequence, except perhaps the $i-j$ one, have started and may be completed.

We now argue by induction.

- Case $n=1$ :

There are only two particles involved, 1 and 2 , so that

$$
\omega_{n} \subset \cup_{l} I_{12, l},
$$

Now, owing to the conservation of the measure by the flow, we may evaluate the measure of $I_{12, l}$ by evaluating the measure of its image by the flow at any time. We choose the time $t_{l}$. At this time $X_{1}, V_{1}$ and $V_{2}$ are undetermined, and $X_{2}$ belongs to the ball centered in $X_{1}$, with radius $3 \varepsilon^{k}$. Thus $\left|I_{12, l}\right|=36 \pi \varepsilon^{3 k}$. From here, we write

$$
\left|\omega_{n}\right| \leq \sum_{l=1}^{M} 36 \pi \varepsilon^{3 k} \leq 108 T \sqrt{3} \varepsilon^{2 k}
$$

This proves the $n=1$ case.

- We now assume that the property is true for all $m<n$.

Then we have

$$
\left|\omega_{n}\right| \leq \sum_{i, j, l}\left|I_{i j, l}\right|
$$

We evaluate the measure of $I_{i j, l}$ by examining the situation at $t=t_{l}$, using again measure conservation. We call $A_{i}=\left\{i, i_{1}, \ldots, i_{m}\right\}$ and $A_{j}=\left\{j, j_{1}, \ldots, j_{n-1-m}\right\}$ the collision sequences to which $i$ and $j$ respectively belong before the $i-j$ collision. $A_{i}$ and $A_{j}$ are two disjoints sets thanks to point $(i i)$ of Theorem 2.5. We call $I_{m}^{(i)}$ the set of initial conditions on $\left\{i, i_{1}, \ldots, i_{m}\right\}$ such that the collision sequence $A_{i}$ happens; at time $t_{l}$, particle $i$ is at a certain position $X_{i}\left(t_{l}\right)=X_{0}$. We call $I_{n-1-m}^{(j), X_{0}}$ the set of initial conditions on $\left\{j, j_{1}, \ldots, j_{n-1-m}\right\}$ such that the collisions of sequence $A_{j}$ happens and particle $j$ is at $t_{l}$ in 
the ball centered in $X_{0}$, of radius $(n+2) \varepsilon^{k}$; using Lemma 4.6 with $\Omega=B\left(X_{0}, 2 \varepsilon^{k}\right) \times \mathbb{R}^{3}$, we know that

$$
\left|I_{n-1-m}^{(j), X_{0}}\right| \leq\left|\omega_{n-1-m}\right| \frac{4(n+2)^{3} \pi}{3} \varepsilon^{3 k}
$$

We then evaluate the measure of $I_{i j, l}$ by

$$
\begin{aligned}
\left|I_{i j, l}\right| & \leq \sum_{m=0}^{n-1} C_{n-1}^{m}\left|I_{m}^{(i)}\right|\left|I_{n-1-m}^{(j), X_{0}}\right| \\
& \leq C \sum_{m=0}^{n-1} C_{n-1}^{m}\left|\omega_{m}\right|\left|\omega_{n-1-m}\right| \varepsilon^{3 k} \\
& \leq C \sum_{m=0}^{n-1} C_{n-1}^{m} a_{m} a_{n-1-m} \varepsilon^{3 k+2 k(n-1)}
\end{aligned}
$$

The last inequality uses the induction assumption. Finally, we have

$$
\begin{aligned}
\left|\omega_{n}\right| & \leq \sum_{i, j=1}^{n} \sum_{l=1}^{M} \sum_{m=0}^{n-1} C_{n-1}^{m} a_{m} a_{n-1-m} \varepsilon^{3 k+2 k(n-1)} \\
& \leq c T \sqrt{n+1} \varepsilon^{-k} n^{2} \sum_{m=0}^{n-1} C_{n-1}^{m} a_{m} a_{n-1-m} \varepsilon^{3 k+2 k(n-1)} \\
& \leq a_{n} \varepsilon^{2 k n}
\end{aligned}
$$

with

$$
a_{n}=c T \sqrt{n+1} n^{2} \sum_{m=0}^{n-1} C_{n-1}^{m} a_{m} a_{n-1-m} .
$$

Notice that the expression for $a_{n}$ is far from optimal and that better bounds could be obtained, this is however enough to complete the proof.

4.2. Proof of points $(i)$ and $(i i)$ of Theorem 2.5. Let us first note that both $(i)$ and $(i i)$ are true for $T=0:(i i)$ obviously there cannot be recollisions as there is no dynamic ; $(i)$ as the set of initial data for which at least three particles are in the same ball of radius $\varepsilon^{k}$ has measure less than $C N^{3} \varepsilon^{6 k}$.

Therefore for any $I \in O$ with $\left|[-1,1]^{2 N} \backslash O\right| \leq C N^{3} \varepsilon^{6 k}$, points $(i)$ and $(i i)$ hold at time 0 .

Now fix $T>0$, and denote by $B$ the set of initial conditions $I$ such that either $(i)$ or $(i i)$ are not true until time $T$. We will show that

$$
|B| \leq C_{T} N^{3} \varepsilon^{5 k}
$$


For any $I \in B$, there exists $t_{0}>0$ such that $(i)$ and (ii) are true until $t_{0}$ and one of them (or both) false at $t_{0}\left(t_{0}\right.$ depends on $\left.I\right)$. This is because both are true at time 0 and because the dynamics is continuous in time.

Notice that all results of subsection 4.1 are true until $t_{0}$. As $k>3$, there exists $n$ independent of $N$ such that asymptotically in $N$

$$
N^{n+1} \varepsilon^{2 k n} \leq N^{3} \varepsilon^{5 k} .
$$

Therefore, it is enough to consider $\tilde{B}=B \backslash O_{n}$ as $\left|O_{n}\right| \leq C_{T} N^{3} \varepsilon^{5 k}$ and for any $I \in \tilde{B}$ there are at most $n-1$ collisions in the dynamics for any sequence of collisions.

In fact we can be a bit more precise and notice that Theorem 2.5 is useless and trivial when $N^{3} \varepsilon^{5 k}$ is not less than 1 . Recalling that $\varepsilon=N^{-1 / 6}$ this implies that it is enough to consider $k \geq 18 / 5$. Consequently, $n=5$ and there are at most 4 collisions (in any sequence) in the dynamics we consider.

We have to study both cases where $(i)$ (respectively $(i i)$ ) is contradicted at time $t_{0}$. Denote by $\tilde{B}_{1} \subset \tilde{B}$ the set of initial conditions $I \in \tilde{B}$ such that there exist $t_{0} \leq T$ and three particles $i, j, k$ with $X_{i}\left(t_{0}\right)$, $X_{j}\left(t_{0}\right)$ and $X_{k}\left(t_{0}\right)$ in the same ball of size $2 \varepsilon^{k}$. Clearly $\tilde{B}_{1}$ contains all initial conditions of $\tilde{B}$ contradicting $(i)$ but is a bit larger (which will be useful for (ii)).

Accordingly denote by $\tilde{B}_{2}$ the set of initial conditions $I \in \tilde{B} \backslash \tilde{B}_{1}$ for which there exists $t_{0}$, and $i$ and $j$ such that for some $t<t_{0}, i R_{t} j$ and $i$ and $j$ collide at $t_{0}$ with $t_{0}$ the beginning of the collision; which means that $\left|X_{i}\left(t_{0}\right)-X_{j}\left(t_{0}\right)\right|=\varepsilon^{k}$ and $\left|X_{i}(t)-X_{j}(t)\right|>\varepsilon^{k}$ for any $t<t_{0}$ but close enough from $t_{0}$. $\tilde{B}_{2}$ contains all initial conditions contradicting (ii) before $(i)$ and of course $\tilde{B}=\tilde{B}_{1} \cup \tilde{B}_{2}$.

4.2.1. Case of $\tilde{B}_{1}:(i)$ is contradicted first. Denote by $\tilde{B}_{1}\left(t_{1}\right)$ the set of initial conditions $I$ such that $I \in \tilde{B}_{1}$ and at $t_{1}$ we have three particles located in the same ball of size $3 \varepsilon^{k}$. As there are $C_{N}^{3}$ possibilities of choosing those three particles, the measure of the set of $X_{1}\left(t_{1}\right), V_{1}\left(t_{1}\right), \ldots X_{N}\left(t_{1}\right), V_{N}\left(t_{1}\right)$ such that three particles are located in the same ball of size $3 \varepsilon^{k}$ at $t_{1}$ is less than

$$
C N^{3} \varepsilon^{6 k} \text {. }
$$

Therefore, since the dynamics preserves volume in the whole phase space, the set $\tilde{B}_{1}\left(t_{1}\right)$ has measure less than $C N^{3} \varepsilon^{6 k}$.

We divide the time interval $[0, T]$ into $M$ intervals $\left[T_{\alpha}, T_{\alpha+1}\right], \alpha=$ $0 \ldots M$, of size $\varepsilon^{k}(n+1)^{-1 / 2}$. If three particles are located in a ball of radius $\varepsilon^{k}$ at $t_{0}$, lemma 4.5 ensures that they are still within a ball of 
radius $6 \varepsilon^{k}$ at some well chosen time $T_{\alpha}$. We have also used that there at most 4 collisions in any sequence before $t_{0}$. So

$$
\tilde{B}_{1}=\bigcup_{\alpha=1}^{M} \tilde{B}_{1}\left(T_{\alpha}\right)
$$

and as $M$ is less than $T \varepsilon^{-k} \sqrt{n+1}$, we finally obtained that

$$
\left|\tilde{B}_{1}\right| \leq \sum_{\alpha=1}^{M}\left|\tilde{B}_{1}\left(T_{\alpha}\right)\right| \leq C M N^{3} \varepsilon^{6 k} \leq C_{T} N^{3} \varepsilon^{5 k},
$$

which is the desired estimate on $\tilde{B}_{1}$.

4.2.2. Case of $\tilde{B}_{2}$ : (ii) is contradicted first. Let us first perform the same subdivision of the time interval $[0, T]$ in $M$ intervals $\left[T_{\alpha}, T_{\alpha+1}\right]$ of size $\varepsilon^{k}(n+1)^{-1 / 2}$ as in the previous paragraph. As the same bound on the velocities applies, we may again assume that $t_{0}$ is one of the $T_{\alpha}$ in the following sense :

Lemma 4.7. There exist $i, j$, $\alpha$ such that $i R_{T_{\alpha}} j$, and $\varepsilon^{k}<\mid X_{i}\left(T_{\alpha}\right)-$ $X_{j}\left(T_{\alpha}\right) \mid \leq 2 \varepsilon^{k}$.

Proof. We use the definition of $\tilde{B}_{2}$ and take $\alpha$ such that $T_{\alpha}=$ $\sup _{\beta}\left\{T_{\beta}<t_{0}\right\}$. Neither $i$ nor $j$ may have any collision with another particle between $T_{\alpha}$ and $t_{0}$. Indeed, suppose the last collision of $i$ between $T_{\alpha}$ and $t_{0}$ is with particle $k$, and ends at $t_{1}$. Between $t_{1}$ and $t_{0}$, the velocities of $i$ and $j$ are bounded by $\sqrt{n+1}$ thanks to lemma 4.4 . Thus, at $t_{1} i, j$ and $k$ would be in a ball of size $2 \varepsilon^{k}$ and the initial condition would belong to $\tilde{B}_{1}$ and thus not $\tilde{B}_{2}$. Note now that since the trajectories of $i$ and $j$ are lines between two collisions, they cannot cross more than once. This implies that $i$ and $j$ do not collide between $T_{\alpha}$ and $t_{0}$. Thus, applying the velocity bound of lemma 4.4, we have $\varepsilon^{k}<\left|X_{i}\left(T_{\alpha}\right)-X_{j}\left(T_{\alpha}\right)\right| \leq 2 \varepsilon^{k}$ and $i R_{T_{\alpha}} j$ (remember that $i R_{t_{0}} j$ ).

Lemma 4.8. For any $\beta \geq 1$ and any two points $x_{i}$ and $x_{f}$ in $[-1-$ $2 T, 1+2 T]^{3}$, denote by $S\left(x_{i}, x_{f}, \beta\right)$ the set of initial conditions $X_{1}(0)$, $X_{2}(0), V_{1}(0), V_{2}(0)$ in $[-1-2 T, 1+2 T]^{6} \times[-2,2]^{6}$ such that (i) $X_{1}(0) \in B\left(x_{i}, 2 \varepsilon^{k}\right)$,

(ii) the two particles 1 and 2 have a collision between time 0 and $T_{\beta}$ (and no collision with any other particles), and

(iii) $X_{1}\left(T_{\beta}\right) \in B\left(x_{f}, 2 \varepsilon^{k}\right)$ or $X_{2}\left(T_{\beta}\right) \in B\left(x_{f}, 2 \varepsilon^{k}\right)$.

Then

$$
\left|S\left(x_{i}, x_{f}, \beta\right)\right| \leq C \frac{\varepsilon^{6 k}}{\beta^{2}}
$$


Note that as the particles have no collision with other particles, the dynamics really involves only them, which is why $S$ contains only the initial conditions for those two particles.

Proof. The dynamics to consider is simply

$$
\begin{aligned}
& \dot{X}_{1}(t)=X_{1}(t), \dot{X}_{2}(t)=V_{2}(t), \\
& \dot{V}_{1}(t)=\frac{1}{N} K_{\varepsilon}\left(X_{1}-X_{2}\right), \quad \dot{V}_{2}=\frac{1}{N} K_{\varepsilon}\left(X_{2}-X_{1}\right),
\end{aligned}
$$

Let us consider $S_{j}\left(x_{i}, x_{f}, \beta\right)$ for $j=1,2$ the subset of $S\left(x_{i}, x_{f}, \beta\right)$ for which $X_{j}\left(T_{\beta}\right) \in B\left(x_{f}, 2 \varepsilon^{k}\right)$ (i.e. (iii) is true for $X_{j}$ ). The set $S$ is the union of $S_{1}$ and $S_{2}$ and it is obviously enough to prove the estimate for those subsets.

Now denote by $\tilde{S}_{j}\left(x_{i}, x_{f}, \beta\right)$ the set of initial conditions $X_{1}(0), X_{2}(0)$, $V_{1}(0), V_{2}(0)$ in $[-1-2 T, 1+2 T]^{6} \times[-3,3]^{6}$ such that again conditions $(i)-($ iii $)$ of 4.8 are satisfied with $X_{j}\left(T_{\beta}\right) \in B\left(x_{f}, 2 \varepsilon^{k}\right)$. The only difference between $\tilde{S}_{j}$ and $S_{j}$ is that $\tilde{S}_{j}$ allows for a larger support in velocity initially.

For any fixed $x_{f}$ and $V$ with $|V| \leq 1$, we have that

$$
S_{j}\left(x_{i}, x_{f}, \beta\right)+(0,0, V, V) \subset \tilde{S}_{j}\left(x_{i}, x_{f}+V T_{\beta}\right) .
$$

This is indeed only galilean invariance : if we add $V$ to the velocities of each particle initially, then they follow the same dynamics with the same $V$ added to their velocities and $V t$ added to their position. Hence the final position $X_{j}\left(T_{\beta}\right)$ is simply shifted by $V T_{\beta}$.

As a consequence for any $x_{f}$ and any $y \in B\left(x_{f}, T_{\beta}\right)$, we have that

$$
\left|\tilde{S}_{j}\left(x_{i}, y, \beta\right)\right| \geq\left|S_{j}\left(x_{i}, x_{f}, \beta\right)\right| \text {. }
$$

Take $L$ points $y^{n}$ in the ball $B\left(x_{f}, T_{\beta}\right)$ such that the distance between any two of them is at least $4 \varepsilon^{k}$. Then clearly

$$
\tilde{S}_{j}\left(x_{i}, y^{n}, \beta\right) \cap \tilde{S}_{j}\left(x_{i}, y^{p}, \beta\right)=\emptyset, \quad \text { if } n \neq p,
$$

as the final position $X_{j}\left(T_{\beta}\right)$ cannot be in both $B\left(y^{n}, 2 \varepsilon^{k}\right)$ and $B\left(y^{p}, 2 \varepsilon^{k}\right)$. Obviously there are at most $L=C \beta^{3}$ such points $y^{n}$ (remember that $T_{\beta}$ is of the order of $\beta \varepsilon^{k}$ ).

On the other hand $U=\bigcup_{n=1}^{L} \tilde{S}_{j}\left(x_{i}, y^{n}, \beta\right)$ contains only those initial data such that $X_{1}(0) \in B\left(x_{i}, 2 \varepsilon^{k}\right)$ and 1 and 2 have a collision between time 0 and $T_{\beta}$. Therefore its measure is at most $C \beta \varepsilon^{6 k}$. Consequently

$$
L \times\left|S_{j}\left(x_{i}, x_{f}, \beta\right)\right| \leq \sum_{n=1}^{L}\left|\tilde{S}_{j}\left(x_{i}, y^{n}, \beta\right)\right|=\left|\bigcup_{n=1}^{L} \tilde{S}_{j}\left(x_{i}, y^{n}, \beta\right)\right| \leq C \beta \varepsilon^{6 k} .
$$


Figure 1. An example of collision sequence when particle $i$ has no collision between $T_{\beta}$ and $T_{\alpha}$; the collision sequence of particles $j$ and $q$ may be more complicated than what is sketched here, in the limit of four or less collisions before $T_{\alpha}$.

So finally, we obtain that

$$
\left|S_{j}\left(x_{i}, x_{f}, \beta\right)\right| \leq C \frac{\varepsilon^{6 k} \beta}{L}=C \frac{\varepsilon^{6 k}}{\beta^{2}} .
$$

As $i$ and $j$ belong to the same sequence before $T_{\alpha}$, there exist two particles $p$ and $q$ (one or both of which could be $i$ or $j$ ) colliding at some time $t_{1}<T_{\alpha}$ ( $t_{1}$ being here the final time of the collision) and such that $p$ and $i$ belong to the same sequence between $t_{1}$ and $T_{\alpha}$ and so do respectively $q$ and $j$.

One of these sequences of collisions contains at least one collision: If not $i$ and $j$ would have one collision at $t_{1}$, another one at $t_{0}$ and none in between, which was already excluded.

On the other hand, one also has at most one collision. Indeed there are at most four collisions before $T_{\alpha}$ and one already occured at $t_{1}$ so there are only three remaining. This is too few to have at least two other collisions for each sequence.

Define $T_{\beta}$ as the smallest of the $T_{\gamma}$ bigger than $t_{1} . p$ and $q$ have at most 3 collisions between $t_{1}$ and $T_{\beta}$, and the $\sqrt{n+1}$ velocity bound applies between the collision, so that

$$
\left|X_{p}\left(T_{\beta}\right)-X_{q}\left(T_{\beta}\right)\right| \leq 6 \varepsilon^{k},
$$

and we may replace $t_{1}$ by $T_{\beta}$.

Now assume that the sequence with $p$ and $i$ between $t_{\beta}$ and $T_{\alpha}$ is the one with the less collisions (and hence 0 or 1 ). Let us consider both cases

- No collision for $i$ in $\left[T_{\beta}, T_{\alpha}\right]$. That means that $i=p$. Accordingly we know that $j \neq q$. Let us denote by $\tilde{B}_{2}^{1}$ the set of initial data such that this occurs and estimate its measure. Fig. 1 gives an example of collision sequence corresponding to this case.

In order to do so, we bound the set of positions and velocities at time $T_{\beta}$ and use Liouville's theorem to deduce a bound on $\tilde{B}_{2}^{1}$. Therefore denote by $C_{1}(\beta)$ the set of corresponding positions and velocities st 
Figure 2. An example of collision sequence when particle $i$ has one collision between $T_{\beta}$ and $T_{\alpha}$; the collision sequence of particles $j$ and $q$ may be more complicated than what is sketched here, in the limit of four or less collisions before $T_{\alpha}$.

time $T_{\beta} X_{n}\left(T_{\beta}\right), V_{n}\left(T_{\beta}\right), 1 \leq n \leq N$. We have that

$$
\left|\tilde{B}_{2}^{1}\right| \leq \sum_{\beta}\left|C_{1}(\beta)\right|
$$

First choose $X_{n}\left(T_{\beta}\right), V_{n}\left(T_{\beta}\right)$ for any $n \neq i$ such that $j$ and $q$ belong to the same sequence of collisions between $T_{\beta}$ and $T_{\alpha}$ (of any number of collisions); Denote by $C(j, q, i, \beta, \alpha)$ the corresponding set. The positions and velocities of all particles except $i$ are known between $T_{\beta}$ and $T_{\alpha}$, as $i$ does not interact with any of them within this time interval.

Once this is done, we must choose $X_{i}\left(T_{\beta}\right)$ in the ball $B\left(X_{q}\left(T_{\beta}\right), \varepsilon^{k}\right)$ and as its trajectory is a line, $V_{i}\left(T_{\beta}\right)$ must lie in the ball centered at $\left(X_{j}\left(T_{\alpha}\right)-X_{i}\left(T_{\beta}\right)\right) /\left(T_{\alpha}-T_{\beta}\right)$, and of radius $\left.\varepsilon^{k} /\left(T_{\alpha}-T_{\beta}\right)\right)$. Therefore the set of corresponding $X_{i}\left(T_{\beta}\right)$ and $V_{i}\left(T_{\beta}\right)$ is of measure less than $C \varepsilon^{3 k}(\alpha-\beta)^{-3}$. Moreover

$$
\begin{aligned}
\left|C_{1}(\beta)\right| & \leq \sum_{i, j, q} \sum_{\alpha} \int_{C(j, q, i, \beta, \alpha)} \int_{X_{i} \in B\left(X_{q}, \varepsilon^{k}\right)} \int_{\left(T_{\alpha}-T_{\beta}\right) V_{i} \in B\left(\left(X_{j}\left(T_{\alpha}\right)-X_{i}\right), \varepsilon^{k}\right)} d V_{i} d X_{i} d X_{1} d V_{1} d V_{N} \\
& \leq C \sum_{i, j, q} \sum_{\alpha} \varepsilon^{6 k}\left(T_{\alpha}-T_{\beta}\right)^{-3}|C(j, q, i, \beta, \alpha)| .
\end{aligned}
$$

The last step is to evaluate the measure of $C(j, q, i, \beta, \alpha)$. As there is at least one collision between $T_{\beta}$ and $T_{\alpha}$, say between particles $k$ and $l$, there exists $\gamma \in[\beta, \alpha]$ such that $k$ and $l$ are in the same ball of size $2 \varepsilon^{k}$. Evaluating the measure of $C(j, q, i, \beta, \alpha)$ by its image by the flow at time $T_{\gamma}$, and summing over all possible $\gamma$, we obtain that $|C(j, q, i, \beta, \alpha)| \leq C \varepsilon^{3 k}(\alpha-\beta)$. Finally, we conclude:

$$
\left|C_{1}(\beta)\right| \leq C N^{3} \varepsilon^{6 k}
$$

and

$$
\left|\tilde{B}_{2}^{1}\right| \leq \sum_{\beta}\left|C_{1}(\beta)\right| \leq C N^{3} \varepsilon^{5 k}
$$

which is the desired estimate.

- One collision for $i$ in $\left[T_{\beta}, T_{\alpha}\right]$. That means that $p=i$ or $p=l$. Fig. 2 gives an example of collision sequence corresponding to this case. 
We follow the same steps as in the previous lemma, and denote by $\tilde{B}_{2}^{2}$ the corresponding set of initial conditions and $C_{2}(\beta)$ the set of $X_{n}\left(T_{\beta}\right), V_{n}\left(T_{\beta}\right)$.

As before we first choose $X_{n}\left(T_{\beta}\right), V_{n}\left(T_{\beta}\right)$ for $n \neq i, l$ in the set $C(j, q, i, l, \beta, \alpha)$, which is such that $j$ and $q$ belong to the same sequence of collisions between $T_{\beta}$ and $T_{\alpha}$. This set is almost exactly $C(j, q, i, \beta, \alpha)$ except that particle 1 is not included.

Then it remains to choose $X_{i}\left(T_{\beta}\right), V_{i}\left(T_{\beta}\right), X_{l}\left(T_{\beta}\right)$ and $V_{l}\left(T_{\beta}\right)$ such that $i$ and $l$ have a collision between $T_{\beta}$ and $T_{\alpha}, X_{i}\left(T_{\alpha}\right)$ belongs to $B\left(X_{j}\left(T_{\alpha}, 2 \varepsilon^{k}\right)\right.$ and $X_{i}\left(T_{\beta}\right)$ or $X_{l}\left(T_{\beta}\right)$ belong to $B\left(X_{q}\left(T_{\beta}\right), 2 \varepsilon^{k}\right)$. This is the set $S\left(X_{q}(\beta), X_{j}(\alpha), \alpha-\beta\right)$ of Lemma 4.8, with the roles of $x_{i}$ and $x_{f}$ exchanged. As the dynamics is reversible, Lemma 4.8 applies. So

$$
\begin{aligned}
\left|C_{2}(\beta)\right| & \leq \sum_{i, j, q} \sum_{\alpha} \int_{C(j, q, i, l, \beta, \alpha)} \int_{S\left(X_{q}, X_{j}(\beta), \alpha-\beta\right)} d V_{i} d X_{i} d V_{l} d X_{l} d X_{1} d V_{1} \ldots d X_{N} d V_{N} \\
& \leq C \sum_{i, j, q, l} \sum_{\alpha} \varepsilon^{6 k}(\alpha-\beta)^{-2}|C(j, q, i, l \beta, \alpha)|
\end{aligned}
$$

according to Lemma 4.8. The estimate for $|C(j, q, i, l, \beta, \alpha)|$ being the same as before, one obtains

$$
\left|\tilde{B}_{2}^{2}\right| \leq C \sum_{\alpha>\beta} \sum_{i, j, q, l} \varepsilon^{9 k}(\alpha-\beta)^{-1} \leq C N^{4} \varepsilon^{8 k}|\ln \varepsilon|,
$$

which is asymptotically less than $N^{3} \varepsilon^{5 k}$ as $N \varepsilon^{3 k}|\ln \varepsilon|$ converges to 0 $\left(N=\varepsilon^{-6}\right.$ and $\left.k>3\right)$. Finally this does imply that

$$
\left|\tilde{B}_{2}\right| \leq\left|\tilde{B}_{2}^{1}\right|+\left|\tilde{B}_{2}^{2}\right| \leq C N^{3} \varepsilon^{5 k}
$$

\section{REFERENCES}

[1] L. Ambrosio, Transport equation and Cauchy problem for BV vector fields. Invent. Math. 158, no. 2, pp 227-260, 2004.

[2] C. Bardos, F. Golse, N. Mauser Weak coupling limit of the $N$-particle Schrödinger equation, Methods and Applications of Analysis 7, pp 275-293, 2000.

[3] C. Bardos, L. Erdös, F. Golse, N. Mauser, H.T. Yau, Derivation of the Schrdinger-Poisson equation from the quantum $N$-body problem. C. R. Math. Acad. Sci. Paris 334, no. 6, pp 515-520, 2002.

[4] W. Braun and K. Hepp, The Vlasov dynamics and its fluctuations in the $1 / N$ limit of interacting particles, Comm. Math. Phys. 56, pp 101-113, 1977.

[5] C. Cercignani, R. Illner and M. Pulvirenti, The mathematical theory of dilute gases, Applied Mathematical Sciences, 106, Springer-Verlag New-York, 1994.

[6] R. L. Dobrushin, Vlasov equations, Funct. Anal. Appl. 13, pp 115-123, 1979. 
[7] J. Goodman, T. Y. Hou and J. Lowengrub, Convergence of the point vortex method for the 2-D Euler equations, Comm. Pure Appl. Math. 43, pp 415-430, 1990.

[8] M. Hauray, On Liouville transport equation with force field in $B V_{\mathrm{loc}}$, Comm. Partial Differential Equations 29, no. 1-2, pp 207-217, 2004.

[9] M. Hauray and P. E.Jabin, N-particles approximation of the Vlasov equation with singular potential, Arch. Ration. Mech. Anal. 183, no. 3, pp 489-524, 2007.

[10] R. Illner and M. Pulvirenti, Global validity of the Boltzmann equation for two and three-dimensional rare gas in vacuum, Comm. Math. Phys. 121, pp 143146, 1989.

[11] P.E. Jabin, F. Otto, Identification of the dilute regime in particle sedimentation, Comm. Math. Phys., 250, pp 415-432, 2004.

[12] O.E. Lanford, Dynamical systems, theory and applications (Battelle Rencontres, Seattle, Wash., 1974), pp. 1-111, Lecture Notes in Phys., Vol. 38, Springer, Berlin, 1975.

[13] O.E. Lanford, On a derivation of the Boltzmann equation. International Conference on Dynamical Systems in Mathematical Physics (Rennes, 1975), pp. 117-137. Asterisque, No. 40, Soc. Math. France, Paris, 1976.

[14] H. Neunzert, J. Wick, Theoretische und numerische Ergebnisse zur nicht linearen Vlasov Gleichung. Numerische Lösung nichtlinearer partieller Differential und Integrodifferentialgleichungen (Tagung, Math. Forschungsinst., Oberwolfach, 1971), pp. 159-185. Lecture Notes in Math., Vol. 267, Springer, Berlin, 1972.

[15] S. Schochet, The weak vorticity formulation of the 2-D Euler equations and concentration-cancellation, Comm. Partial Differential Equations, 20, pp 10771104, 1995.

[16] S. Schochet, The point-vortex method for periodic weak solutions of the 2-D Euler equations, Comm. Pure Appl. Math., 49, pp 911-965, 1996.

[17] H. Spohn, Large scale dynamics of interacting particles, Springer-Verlag Berlin, 1991.

[18] H.D. Victory, jr., E.J. Allen, The convergence theory of particle-in-cell methods for multidimensional Vlasov-Poisson systems, SIAM J. Numer. Anal., 28, pp 1207-1241, 1991.

[19] S. Wollman, On the approximation of the Vlasov-Poisson system by particles methods, SIAM J. Numer. Anal., 37, pp 1369-1398, 2000.

Laboratoire J. A. Dieudonné, UMR CNRS 6621, Université de NiceSophia Antipolis, Parc Valrose, 06108 Nice, France. 